\title{
Two variable higher-order central Fubini polynomials
}

Taekyun Kim ${ }^{1}$, Dae San Kim², Gwan-Woo Jang ${ }^{1}$ and Dojin Kim ${ }^{3 *}$

This article is dedicated to Professor Gradimir V. Milovanovic on the occasion of his 70th anniversary.

${ }^{*}$ Correspondence:

kimdojin@pusan.ac.kr

${ }^{3}$ Department of Mathematics,

Pusan National University, Busan, Republic of Korea

Full list of author information is

available at the end of the article

\begin{abstract}
Recently, the central Fubini polynomials were introduced in connection with central factorial numbers of the second kind. In this paper, we consider two variable higher-order central Fubini polynomials as a 'central analogue' of two variable higher-order Fubini polynomials. We investigate some properties, identities, and recurrence relations for these polynomials by making use of generating functions and umbral calculus. In particular, we obtain various expressions for the two variable higher-order central Fubini polynomials and express them in terms of some families of special polynomials and vice versa.
\end{abstract}

Keywords: Two variable higher-order central Fubini polynomials; Two variable central Fubini polynomials; Umbral calculus

\section{Introduction}

For $n \in \mathbb{N} \cup\{0\}$, the central factorial $x^{[n]}$ is defined as

$$
x^{[0]}=1, \quad x^{[n]}=x\left(x+\frac{n}{2}-1\right)\left(x+\frac{n}{2}-2\right) \cdots\left(x-\frac{n}{2}+1\right) \quad(n \geq 1) .
$$

As is well known, the central factorial numbers of the second kind $T(n, k)(n, k \geq 0)$ are defined by

$$
x^{n}=\sum_{k=0}^{n} T(n, k) x^{[k]} \quad(n \geq 0)(\text { see }[7-9,11,13,16-18]) .
$$

From (2), we can derive the following generating function for $T(n, k)$ :

$$
\frac{1}{k !}\left(e^{\frac{t}{2}}-e^{-\frac{t}{2}}\right)^{k}=\sum_{n=k}^{\infty} T(n, k) \frac{t^{n}}{n !} \quad(k \geq 0)(\text { see }[7-9,11-13,16-18]) .
$$

It is known that the two variable Fubini polynomials $F_{n}^{(r)}(x ; y)$ of order $r$ are defined by

$$
\left(\frac{1}{1-y\left(e^{t}-1\right)}\right)^{r} e^{x t}=\sum_{n=0}^{\infty} F_{n}^{(r)}(x ; y) \frac{t^{n}}{n !} \quad(\text { see }[1,2,4-6,14])
$$

where $r$ is a positive integer.

(c) The Author(s) 2019. This article is distributed under the terms of the Creative Commons Attribution 4.0 International License (http://creativecommons.org/licenses/by/4.0/), which permits unrestricted use, distribution, and reproduction in any medium, provided you give appropriate credit to the original author(s) and the source, provide a link to the Creative Commons license, and indicate if changes were made. 
In particular, if $r=1$, then $F_{n}(x ; y)=F_{n}^{(1)}(x ; y)$ are called two variable Fubini polynomials. For $x=0, F_{n}^{(r)}(y)=F_{n}^{(r)}(0 ; y)$ and $F_{n}^{(r)}=F_{n}^{(r)}(1)=F_{n}^{(r)}(0 ; 1)$ are respectively called the Fubini polynomials of order $r$ and the Fubini numbers of order $r$. Further, in the special case of $y=1, F_{n}^{(r)}(x ; 1)$ are the ordered Bell polynomials of order $r$. Recently, the central Fubini polynomials have been defined by

$$
\frac{1}{1-x\left(e^{\frac{t}{2}}-e^{-\frac{t}{2}}\right)}=\frac{1}{1-2 x \sinh \left(\frac{t}{2}\right)}=\sum_{n=0}^{\infty} F_{n, c}(x) \frac{t^{n}}{n !} \quad(\text { see }[8]) .
$$

From (5), one can see that

$$
F_{n, c}(x)=\sum_{k=0}^{n} k ! T(n, k) x^{k} \quad(n \geq 0)(\text { see }[8]) .
$$

Next, we will quickly review very basics of umbral calculus. Let $\mathbb{C}$ be the field of complex numbers, and let

$$
\mathcal{F}:=\left\{f(t)=\sum_{k=0}^{\infty} a_{k} \frac{t^{k}}{k !} \mid a_{k} \in \mathbb{C}\right\} .
$$

We set $\mathbb{P}=\mathbb{C}[x]$ and define $\mathbb{P}^{*}$ by the vector space of all linear functionals on $\mathbb{P}$. For any given $L \in \mathbb{P}^{*}$ and $p(x) \in \mathbb{P}$, throughout the paper, $\langle L \mid p(x)\rangle$ represents the action of the linear functional $L$ on $p(x)$. For $f(t)=\sum_{k=0}^{\infty} a_{k} \frac{t^{k}}{k !} \in \mathcal{F},\langle f(t) \mid \cdot\rangle$ denotes the linear functional on $\mathbb{P}$ given by

$$
\left\langle f(t) \mid x^{n}\right\rangle=a_{n} \quad(n \geq 0)(\text { see }[10,15]) .
$$

For $L \in \mathbb{P}^{*}$, let $f_{L}(t)=\sum_{k=0}^{\infty}\left\langle L \mid x^{k}\right\rangle \frac{t^{k}}{k !} \in \mathcal{F}$. Then we have $\left\langle f_{L}(t) \mid x^{n}\right\rangle=\left\langle L \mid x^{n}\right\rangle,(n \geq 0)$, and the map $L \longmapsto f_{L}(t)$ is a vector space isomorphism from $\mathbb{P}^{*}$ to $\mathcal{F}$. Thus, $\mathcal{F}$ may be viewed as the vector space of all linear functionals on $\mathbb{P}$ as well as the algebra of formal power series in $t$. Hence, an element $f(t) \in \mathcal{F}$ will be thought of as both a formal power series and a linear functional on $\mathbb{P}$. $\mathcal{F}$ is called the umbral algebra, the study of which is the umbral calculus.

The order $O(f(t))$ of $f(t)(\neq 0) \in \mathcal{F}$ is the smallest integer $k$ such that the coefficient of $t^{k}$ does not vanish. For $f(t), g(t) \in \mathcal{F}$ with $O(g(t))=0, O(f(t))=1$, there exists a unique sequence of polynomials $S_{n}(x)\left(\operatorname{deg} S_{n}(x)=n\right)$ such that

$$
\left\langle g(t)(f(t))^{k} \mid S_{n}(x)\right\rangle=n ! \delta_{n, k} \quad(n, k \geq 0)(\text { see [15]), }
$$

where $\delta_{n, k}$ is the Kronecker symbol. Such a sequence is called the Sheffer sequence for $(g(t), f(t))$, which is denoted by $S_{n}(x) \sim(g(t), f(t))$. It is known that $S_{n}(x) \sim(g(t), f(t))$ if and only if

$$
\frac{1}{g(\bar{f}(t))} e^{x \bar{f}(t)}=\sum_{n=0}^{\infty} S_{n}(x) \frac{t^{n}}{n !} \quad(\text { see }[3,10,15]),
$$


where $\bar{f}(t)$ is the compositional inverse of $f(t)$ satisfying $f(\bar{f}(t))=\bar{f}(f(t))=t$. For $S_{n}(x) \sim$ $(g(t), f(t))$, we have the following Sheffer identity:

$$
S_{n}(x+y)=\sum_{k=0}^{n}\left(\begin{array}{l}
n \\
k
\end{array}\right) S_{k}(x) P_{n-k}(y)
$$

where $P_{n}(x)=g(t) S_{n}(x) \sim(1, f(t))$ (see [15]).

For any $h(t) \in \mathcal{F}, p(x) \in \mathbb{P}$, we have

$$
\langle h(t) \mid x p(x)\rangle=\left\langle\partial_{t} h(t) \mid p(x)\right\rangle \quad(\text { see [15]). }
$$

Let $S_{n}(x) \sim(g(t), f(t))$ and $r_{n}(x) \sim(h(t), l(t))$. Then we have

$$
S_{n}(x)=\sum_{k=0}^{n} C_{n, k} r_{k}(x)
$$

where

$$
C_{n, k}=\frac{1}{k !}\left\langle\frac{h(\bar{f}(t))}{g(\bar{f}(t))}(l(\bar{f}(t)))^{k} \mid x^{n}\right\rangle \quad(\text { see }[3,10,15]) .
$$

In the forthcoming section, we will consider two variable higher-order central Fubini polynomials as a 'central analogue' of two variable higher-order Fubini polynomials. We introduce some properties and present several identities and recurrence relations for these polynomials by making use of generating functions and umbral calculus. Further, we show various expressions for the two variable higher-order central Fubini polynomials and express them in terms of some families of special polynomials and vice versa in the following section.

\section{Two variable higher-order central Fubini polynomials}

In view of (4), we consider the two variable higher-order central Fubini polynomials which are given by

$$
\left(\frac{1}{1-y\left(e^{\frac{t}{2}}-e^{-\frac{t}{2}}\right)}\right)^{r} e^{x t}=\sum_{n=0}^{\infty} F_{n, c}^{(r)}(x ; y) \frac{t^{n}}{n !}
$$

where $r$ is a positive integer. Here, in this paper, $y$ will be an arbitrary but fixed real number so that $F_{n, c}^{(r)}(x ; y)$ are polynomials in $x$ for each fixed $y$.

From (9) and (14), we note that

$$
F_{n, c}^{(r)}(x ; y) \sim\left(\left(1-y\left(e^{\frac{t}{2}}-e^{-\frac{t}{2}}\right)\right)^{r}, t\right) .
$$

When $r=1, F_{n, c}^{(1)}(x ; y)=F_{n, c}(x ; y)$ are called two variable central Fubini polynomials and $F_{n, c}^{(r)}(y)=F_{n, c}^{(r)}(0 ; y)$ are called central Fubini polynomials of order $r$. When $r=1, F_{n, c}(y)=$ 
$F_{n, c}^{(1)}(y)$ are called the central Fubini polynomials. From (14), we note that

$$
\begin{aligned}
\sum_{n=0}^{\infty} F_{n, c}^{(r)}(x ; y) \frac{t^{n}}{n !} & =\left(\sum_{l=0}^{\infty} F_{l, c}^{(r)}(y) \frac{t^{l}}{l !}\right)\left(\sum_{m=0}^{\infty} \frac{x^{m}}{m !} t^{m}\right) \\
& =\sum_{n=0}^{\infty}\left(\sum_{l=0}^{n}\left(\begin{array}{l}
n \\
l
\end{array}\right) F_{l, c}^{(r)}(y) x^{n-l}\right) \frac{t^{n}}{n !} .
\end{aligned}
$$

Thus, by comparing the coefficients on both sides of (16), we get

$$
F_{n, c}^{(r)}(x ; y)=\sum_{l=0}^{n}\left(\begin{array}{l}
n \\
l
\end{array}\right) F_{l, c}^{(r)}(y) x^{n-l} \quad(n \geq 0) .
$$

By (17), we easily get $\frac{d}{d x} F_{n, c}^{(r)}(x ; y)=n F_{n-1, c}^{(r)}(x ; y),(n \in \mathbb{N})$. From (14), we note that

$$
\begin{aligned}
\sum_{n=0}^{\infty} F_{n, c}^{(r)}(y) \frac{t^{n}}{n !} & =\left(1-y\left(e^{\frac{t}{2}}-e^{-\frac{t}{2}}\right)\right)^{-r} \\
& =\sum_{k=0}^{\infty}\left(\begin{array}{c}
r+k-1 \\
k
\end{array}\right) y^{k} k ! \frac{1}{k !}\left(e^{\frac{t}{2}}-e^{-\frac{t}{2}}\right)^{k} \\
& =\sum_{k=0}^{\infty}\left(\begin{array}{c}
r+k-1 \\
k
\end{array}\right) k ! y^{k} \sum_{n=k}^{\infty} T(n, k) \frac{t^{n}}{n !} \\
& =\sum_{n=0}^{\infty}\left(\sum_{k=0}^{n}\left(\begin{array}{c}
r+k-1 \\
k
\end{array}\right) k ! y^{k} T(n, k)\right) \frac{t^{n}}{n !} .
\end{aligned}
$$

Comparing the coefficients on both sides of (18), we have the following theorem.

Theorem 1 For $n \geq 0$, we have

$$
F_{n, c}^{(r)}(y)=\sum_{k=0}^{n}\left(\begin{array}{c}
r+k-1 \\
k
\end{array}\right) k ! y^{k} T(n, k)
$$

The ordered central Bell numbers of order $r$ are defined by the generating function

$$
\left(\frac{1}{1-\left(e^{\frac{t}{2}}-e^{-\frac{t}{2}}\right)}\right)^{r}=\left(\frac{1}{1-2 \sinh \left(\frac{t}{2}\right)}\right)^{r}=\sum_{n=0}^{\infty} b_{n, c}^{(r)} \frac{t^{n}}{n !} .
$$

Corollary 1 For $n \geq 0$, we have

$$
b_{n, c}^{(r)}=\sum_{k=0}^{n}\left(\begin{array}{c}
r+k-1 \\
k
\end{array}\right) k ! T(n, k)
$$

Remark 1 When $r=1, b_{n, c}=b_{n, c}^{(1)}$ are called the ordered central Bell numbers. Note that

$$
b_{n, c}=\sum_{k=0}^{n} k ! T(n, k) \quad(n \geq 0)
$$


By (14), we get

$$
\begin{aligned}
\sum_{n=0}^{\infty} F_{n, c}^{(r)}(y) \frac{t^{n}}{n !} & =\left(\frac{1}{1-y\left(e^{\frac{t}{2}}-e^{-\frac{t}{2}}\right)}\right)^{r} \\
& =\sum_{m=0}^{\infty}\left(\begin{array}{c}
r+m-1 \\
m
\end{array}\right) y^{m}\left(e^{\frac{t}{2}}-e^{-\frac{t}{2}}\right)^{m} \\
& =\sum_{m=0}^{\infty}\left(\begin{array}{c}
r+m-1 \\
m
\end{array}\right) y^{m} \sum_{l=0}^{m}\left(\begin{array}{c}
m \\
l
\end{array}\right)(-1)^{m-l} e^{\left(l-\frac{m}{2}\right) t} \\
& =\sum_{n=0}^{\infty}\left(\sum_{m=0}^{\infty}\left(\begin{array}{c}
r+m-1 \\
m
\end{array}\right) y^{m} \delta^{m} 0^{n}\right) \frac{t^{n}}{n !},
\end{aligned}
$$

where $\delta$ is the central difference operator given by

$$
\delta f(x)=f(x+1 / 2)-f(x-1 / 2) .
$$

Therefore, by (20), we obtain the following theorem.

Theorem 2 For $n \geq 0$, we have

$$
F_{n, c}^{(r)}(y)=\sum_{m=0}^{\infty}\left(\begin{array}{c}
r+m-1 \\
m
\end{array}\right) y^{m} \delta^{m} 0^{n}
$$

where $\delta f$ is as in (21). In particular,

$$
b_{n, c}^{(r)}=\sum_{m=0}^{\infty}\left(\begin{array}{c}
r+m-1 \\
m
\end{array}\right) \delta^{m} 0^{n} .
$$

From (14), we can derive the following equation (22):

$$
\begin{aligned}
\sum_{n=0}^{\infty} F_{n, c}^{(r)}(x ; y) \frac{t^{n}}{n !} & =\left(\frac{1}{1-y\left(e^{\frac{t}{2}}-e^{-\frac{t}{2}}\right)}\right)^{r} e^{x t} \\
& =\left(\sum_{l=0}^{\infty} F_{l, c}^{(r)}(y) \frac{t^{l}}{l !}\right)\left(\sum_{m=0}^{\infty} x^{m} \frac{t^{m}}{m !}\right) \\
& =\sum_{n=0}^{\infty}\left(\sum_{l=0}^{n}\left(\begin{array}{l}
n \\
l
\end{array}\right) F_{l, c}^{(r)}(y) x^{n-l}\right) \frac{t^{n}}{n !} .
\end{aligned}
$$

Comparing the coefficients on both sides of (22), the following theorem is obtained.

Theorem 3 For $n \geq 0$, we have

$$
F_{n, c}^{(r)}(x ; y)=\sum_{l=0}^{n} \sum_{k=0}^{l}\left(\begin{array}{l}
n \\
l
\end{array}\right)\left(\begin{array}{c}
r+k-1 \\
k
\end{array}\right) k ! T(l, k) x^{n-l} y^{k} .
$$


In particular,

$$
F_{n, c}^{(r)}(y)=\sum_{k=0}^{n}\left(\begin{array}{c}
r+k-1 \\
k
\end{array}\right) k ! T(n, k) y^{k} .
$$

Remark 2 From (14), we note that

$$
F_{n, c}^{(r)}\left(x_{1}+x_{2} ; y\right)=\sum_{m=0}^{n}\left(\begin{array}{l}
n \\
m
\end{array}\right) F_{m, c}^{(r)}\left(x_{1} ; y\right) x_{2}^{n-m} \quad(n \geq 0) .
$$

From $r \in \mathbb{N}$ with $r \geq 2$, we have

$$
\begin{aligned}
\left(\frac{1}{1-y\left(e^{\frac{t}{2}}-e^{-\frac{t}{2}}\right)}\right)^{r} & =\left(\frac{1}{1-y\left(e^{\frac{t}{2}}-e^{-\frac{t}{2}}\right)}\right)^{r-1}\left(\frac{1}{1-y\left(e^{\frac{t}{2}}-e^{-\frac{t}{2}}\right)}\right) \\
& =\left(\sum_{m=0}^{\infty} F_{m, c}^{(r-1)}(y) \frac{t^{m}}{m !}\right)\left(\sum_{l=0}^{\infty} F_{l, c}(y) \frac{t^{l}}{l !}\right) \\
& =\sum_{n=0}^{\infty}\left(\sum_{m=0}^{n}\left(\begin{array}{c}
n \\
m
\end{array}\right) F_{m, c}^{(r-1)}(y) F_{n-m, c}(y)\right) \frac{t^{n}}{n !} .
\end{aligned}
$$

Therefore, by (14) and (24), we obtain the following convolution formula.

Theorem 4 For $r \in \mathbb{N}$ with $r \geq 2$ and $n \geq 0$, we have

$$
F_{n, c}^{(r)}(y)=\sum_{m=0}^{n}\left(\begin{array}{l}
n \\
m
\end{array}\right) F_{m, c}^{(r-1)}(y) F_{n-m, c}(y) .
$$

Now, we observe that

$$
\begin{aligned}
\left(1-y\left(e^{\frac{t}{2}}-e^{-\frac{t}{2}}\right)\right)^{r} & =\sum_{l=0}^{\infty}\left(\begin{array}{l}
r \\
l
\end{array}\right) l !(-y)^{l} \frac{1}{l !}\left(e^{\frac{t}{2}}-e^{-\frac{t}{2}}\right)^{l} \\
& =\sum_{k=0}^{\infty}\left(\sum_{l=0}^{k}\left(\begin{array}{l}
r \\
l
\end{array}\right)(-y)^{l} l ! T(k, l)\right) \frac{t^{k}}{k !} .
\end{aligned}
$$

From (14) and (25), we have

$$
\begin{aligned}
\sum_{n=0}^{\infty} x^{n} \frac{t^{n}}{n !} & =\sum_{k=0}^{\infty}\left(\sum_{l=0}^{k}\left(\begin{array}{l}
r \\
l
\end{array}\right)(-y) l l ! T(k, l)\right) \frac{t^{k}}{k !} \sum_{m=0}^{\infty} F_{m, c}^{(r)}(x ; y) \frac{t^{m}}{m !} \\
& =\sum_{n=0}^{\infty}\left(\sum_{k=0}^{n}\left(\begin{array}{l}
n \\
k
\end{array}\right) \sum_{l=0}^{k}\left(\begin{array}{l}
r \\
l
\end{array}\right)(-y) l l ! T(k, l) F_{n-k, c}^{(r)}(x ; y)\right) \frac{t^{n}}{n !} .
\end{aligned}
$$

Therefore, by (26), we obtain the following theorem.

Theorem 5 For $n \geq 0$, we have

$$
x^{n}=\sum_{k=0}^{n} \sum_{l=0}^{n-k}\left(\begin{array}{l}
n \\
k
\end{array}\right)\left(\begin{array}{l}
r \\
l
\end{array}\right)(-y)^{l} l ! T(n-k, l) F_{k, c}^{(r)}(x ; y) .
$$


In particular, $n \in \mathbb{N}$,

$$
F_{n, c}^{(r)}(y)=-\sum_{k=0}^{n-1} \sum_{l=0}^{n-k}\left(\begin{array}{l}
n \\
k
\end{array}\right)\left(\begin{array}{l}
r \\
l
\end{array}\right)(-y)^{l} l ! T(n-k, l) F_{k, c}^{(r)}(y) .
$$

From (4) and (9), we note that

$$
F_{n, c}^{(r)}(x ; y) \sim\left(\left(1-y\left(e^{\frac{t}{2}}-e^{-\frac{t}{2}}\right)\right)^{r}, t\right) \quad(n \in \mathbb{N} \cup\{0\}) .
$$

For $n \in \mathbb{N}$, by (11), we get

$$
\begin{aligned}
F_{n, c}^{(r)}(z ; y) & =\left\langle\frac{1}{\left(1-y\left(e^{\frac{t}{2}}-e^{-\frac{t}{2}}\right)\right)^{r}} e^{z t} \mid x^{n}\right\rangle \\
& =\left\langle\left(\partial_{t} \frac{1}{\left(1-y\left(e^{\frac{t}{2}}-e^{-\frac{t}{2}}\right)\right)^{r}}\right) e^{z t} \mid x^{n-1}\right\rangle+\left\langle\frac{1}{\left(1-y\left(e^{\frac{t}{2}}-e^{-\frac{t}{2}}\right)\right)^{r}}\left(\partial_{t} e^{z t}\right) \mid x^{n-1}\right\rangle
\end{aligned}
$$

It is easy to show that

$$
\left\langle\left(\partial_{t}\left(1-y\left(e^{\frac{t}{2}}-e^{-\frac{t}{2}}\right)\right)^{-r}\right) e^{z t} \mid x^{n-1}\right\rangle=r y\left(\frac{1}{2} F_{n-1, c}^{(r+1)}\left(z+\frac{1}{2} ; y\right)+\frac{1}{2} F_{n-1, c}^{(r+1)}\left(z-\frac{1}{2} ; y\right)\right) .
$$

Clearly, the second term of (28) is $z F_{n-1, c}^{(r)}(z ; y)$.

Therefore, we obtain the following theorem.

Theorem 6 For $n \geq 0$, we have

$$
F_{n+1, c}^{(r)}(x ; y)=x F_{n, c}^{(r)}(x ; y)+\frac{r}{2} y\left(F_{n, c}^{(r+1)}\left(x+\frac{1}{2} ; y\right)+F_{n, c}^{(r+1)}\left(x-\frac{1}{2} ; y\right)\right)
$$

and

$$
F_{n+1, c}^{(r)}(y)=\frac{r}{2} y\left(F_{n, c}^{(r+1)}\left(\frac{1}{2} ; y\right)+F_{n, c}^{(r+1)}\left(-\frac{1}{2} ; y\right)\right) .
$$

Now, we will express the two variable higher-order central Fubini polynomials $F_{n, c}^{(r)}(x ; y)$ as linear combinations of some well-known special polynomials.

We first recall that

$$
F_{n, c}^{(r)}(x ; y) \sim\left(\left(1-y\left(e^{\frac{t}{2}}-e^{-\frac{t}{2}}\right)\right)^{r}, t\right) .
$$

If we let

$$
F_{n, c}^{(r)}(x ; y)=\sum_{m=0}^{\infty} C_{n, m} S_{m}(x)
$$


where $S_{n}(x) \sim(h(t), l(t))$. Then, from (12) and (13), we see that

$$
\begin{aligned}
C_{n, m} & =\frac{1}{m !}\left\langle\frac{h(t)}{\left(1-y\left(e^{\frac{t}{2}}-e^{-\frac{t}{2}}\right)\right)^{r}}(l(t))^{m} \mid x^{n}\right\rangle \\
& =\frac{1}{m !}\left\langle h(t)(l(t))^{m} \mid \frac{1}{\left(1-y\left(e^{\frac{t}{2}}-e^{-\frac{t}{2}}\right)\right)^{r}} x^{n}\right\rangle \\
& =\frac{1}{m !}\left\langle h(t)(l(t))^{m} \mid F_{n, c}^{(r)}(x ; y)\right\rangle .
\end{aligned}
$$

Therefore, the following theorem can be established.

Theorem 7 Let $S_{n}(x) \sim(h(t), l(t))$ for $n \geq 0$, then we have

$$
F_{n, c}^{(r)}(x ; y)=\sum_{m=0}^{n} C_{n, m} S_{m}(x),
$$

where

$$
C_{n, m}=\frac{1}{m !}\left\langle h(t) l(t)^{m} \mid F_{n, c}^{(r)}(x ; y)\right\rangle .
$$

Let $B_{n}(x)(n \geq 0)$ be the ordinary Bernoulli polynomials given by

$$
\frac{t}{e^{t}-1} e^{x t}=\sum_{n=0}^{\infty} B_{n}(x) \frac{t^{n}}{n !} .
$$

Note that $B_{n}(x) \sim\left(\frac{e^{t}-1}{t}, t\right)$.

Assume that

$$
F_{n, c}^{(r)}(x ; y)=\sum_{m=0}^{n} C_{n, m} B_{m}(x)
$$

where

$$
\begin{aligned}
C_{n, m} & =\frac{1}{m !}\left\langle\frac{e^{t}-1}{t} \mid t^{m} F_{n, c}^{(r)}(x ; y)\right\rangle \\
& =\left(\begin{array}{c}
n \\
m
\end{array}\right)\left\langle\frac{e^{t}-1}{t} \mid F_{n-m, c}^{(r)}(x ; y)\right\rangle \\
& =\left(\begin{array}{c}
n \\
m
\end{array}\right) \int_{0}^{1} F_{n-m, c}^{(r)}(u ; y) d u \\
& =\left(\begin{array}{c}
n \\
m
\end{array}\right) \frac{1}{n-m+1}\left(F_{n-m+1, c}^{(r)}(1 ; y)-F_{n-m+1, c}^{(r)}(y)\right) \\
& =\frac{1}{n+1}\left(\begin{array}{c}
n+1 \\
m
\end{array}\right)\left(F_{n-m+1, c}^{(r)}(1 ; y)-F_{n-m+1, c}^{(r)}(y)\right) .
\end{aligned}
$$

Therefore, by (33), we obtain the following theorem. 
Theorem 8 For $n \geq 0$, we have

$$
F_{n, c}^{(r)}(x ; y)=\frac{1}{n+1} \sum_{m=0}^{n}\left(\begin{array}{c}
n+1 \\
m
\end{array}\right)\left(F_{n-m+1, c}^{(r)}(1 ; y)-F_{n-m+1, c}^{(r)}(y)\right) B_{m}(x) .
$$

The falling factorial sequence is defined by

$$
(x)_{0}=1, \quad(x)_{n}=x(x-1)(x-2) \cdots(x-n+1) \quad(n \geq 1) .
$$

Note that the generating function of $(x)_{n}$ is given by

$$
(1+t)^{x}=\sum_{n=0}^{\infty}(x)_{n} \frac{t^{n}}{n !}
$$

By (34), we get $(x)_{n} \sim\left(1, e^{t}-1\right)$.

Assume that

$$
F_{n, c}^{(r)}(x ; y)=\sum_{n=0}^{m} C_{n, m}(x)_{m}
$$

Then, by Theorem 7, we have

$$
\begin{aligned}
C_{n, m} & =\frac{1}{m !}\left\langle\left(e^{t}-1\right)^{m} \mid F_{n, c}^{(r)}(x ; y)\right\rangle \\
& =\left\langle\frac{1}{m !}\left(e^{t}-1\right)^{m} \mid F_{n, c}^{(r)}(x ; y)\right\rangle \\
& =\sum_{k=m}^{\infty} S_{2}(k, m) \frac{1}{k !}\left\langle t^{k} \mid F_{n, c}^{(r)}(x ; y)\right\rangle \\
& =\sum_{k=m}^{n} S_{2}(k, m)\left(\begin{array}{l}
n \\
k
\end{array}\right) F_{n-k, c}^{(r)}(y),
\end{aligned}
$$

where $S_{2}(n, k)$ are the numbers of the second kind given by

$$
x^{n}=\sum_{k=0}^{n} S_{2}(n, k)(x)_{k} \quad(n \geq 0) .
$$

Therefore, we obtain the following theorem.

Theorem 9 For $n \geq 0$, we have

$$
F_{n, c}^{(r)}(x ; y)=\sum_{m=0}^{n} \sum_{k=m}^{n}\left(\begin{array}{l}
n \\
k
\end{array}\right) S_{2}(k, m) F_{n-k, c}^{(r)}(y)(x)_{m}
$$

It is well known that the Bell polynomials are defined by the generating function

$$
e^{x\left(e^{t}-1\right)}=\sum_{n=0}^{\infty} \operatorname{Bel}_{n}(x) \frac{t^{n}}{n !}
$$


Thus, by (37), we get

$$
\operatorname{Bel}_{n}(x) \sim(1, \log (1+t)) \quad(n \geq 0)
$$

Assume that

$$
F_{n, c}^{(r)}(x ; y)=\sum_{m=0}^{n} C_{n, m} \operatorname{Bel}_{m}(x) .
$$

By Theorem 7, we get

$$
\begin{aligned}
C_{n, m} & =\left\langle\frac{1}{m !}(\log (1+t))^{m} \mid F_{n, c}^{(r)}(x ; y)\right\rangle \\
& =\sum_{k=m}^{\infty} S_{1}(k, m) \frac{1}{k !}\left\langle t^{k} \mid F_{n, c}^{(r)}(x ; y)\right\rangle \\
& =\sum_{k=m}^{n}\left(\begin{array}{l}
n \\
k
\end{array}\right) S_{1}(k, m) F_{n-k, c}^{(r)}(y),
\end{aligned}
$$

where $S_{1}(n, k)$ are the Stirling numbers of the first kind defined by

$$
(x)_{n}=\sum_{k=0}^{n} S_{1}(n, k) x^{k} \quad(n \geq 0) .
$$

Therefore, we obtain the following theorem.

Theorem 10 For $n \geq 0$, we have

$$
F_{n, c}^{(r)}(x ; y)=\sum_{m=0}^{n} \sum_{k=m}^{n}\left(\begin{array}{l}
n \\
k
\end{array}\right) S_{1}(k, m) F_{n-k, c}^{(r)}(y) \operatorname{Bel}_{m}(x) .
$$

Let $p(x) \in \mathbb{C}[x]$ be a polynomial of degree $\leq n$. Then we can write

$$
p(x)=\sum_{m=0}^{n} a_{m} F_{m, c}^{(r)}(x ; y) \quad \text { for } a_{m} \in \mathbb{C} .
$$

We observe that

$$
\begin{aligned}
\left\langle\left(1-y\left(e^{\frac{t}{2}}-e^{-\frac{t}{2}}\right)\right)^{r} t^{m} \mid p(x)\right\rangle & =\sum_{l=0}^{n} a_{l}\left\langle\left(1-y\left(e^{\frac{t}{2}}-e^{-\frac{t}{2}}\right)\right)^{r} t^{m} \mid F_{l, c}^{(r)}(x ; y)\right\rangle \\
& =\sum_{l=0}^{n} a_{l} l ! \delta_{m, l} \\
& =m ! a_{m} .
\end{aligned}
$$


Thus, by (42), we get

$$
\begin{aligned}
a_{m} & =\frac{1}{m !}\left\langle\left(1-y\left(e^{\frac{t}{2}}-e^{-\frac{t}{2}}\right)\right)^{r} t^{m} \mid p(x)\right\rangle \\
& =\frac{1}{m !}\left\langle\sum_{l=0}^{\infty}\left(\begin{array}{l}
r \\
l
\end{array}\right) l !(-y)^{l} \frac{1}{l !}\left(e^{\frac{t}{2}}-e^{-\frac{t}{2}}\right)^{l} \mid t^{m} p(x)\right\rangle \\
& =\frac{1}{m !}\left\langle\sum_{l=0}^{\infty}\left(\begin{array}{l}
r \\
l
\end{array}\right) l !(-y)^{l} \sum_{k=l}^{\infty} T(k, l) \frac{t^{k}}{k !} \mid t^{m} p(x)\right\rangle \\
& =\frac{1}{m !}\left\langle\sum_{k=0}^{\infty} \frac{1}{k !} \sum_{l=0}^{k}\left(\begin{array}{l}
r \\
l
\end{array}\right) l !(-y)^{l} T(k, l) \mid t^{m+k} p(x)\right\rangle \\
& =\frac{1}{m !} \sum_{k=0}^{n-m} \frac{1}{k !} \sum_{l=0}^{k}\left(\begin{array}{l}
r \\
l
\end{array}\right) l !(-y)^{l} T(k, l)\left\langle 1 \mid t^{m+k} p(x)\right\rangle .
\end{aligned}
$$

Therefore, we obtain the following theorem.

Theorem 11 For $p(x) \in \mathbb{C}[x]$ with $\operatorname{deg} p(x) \leq n$, we have

$$
p(x)=\sum_{m=0}^{n} a_{m} F_{m, c}^{(r)}(x ; y) \quad\left(a_{m} \in \mathbb{C}\right)
$$

where

$$
a_{m}=\frac{1}{m !} \sum_{k=0}^{n-m} \frac{1}{k !} \sum_{l=0}^{k}\left(\begin{array}{l}
r \\
l
\end{array}\right) l !(-y)^{l} T(k, l)\left\langle 1 \mid t^{m+k} p(x)\right\rangle .
$$

For example, let $p(x)=B_{n}(x)(n \geq 0)$. Then we have

$$
B_{n}(x)=\sum_{m=0}^{n} a_{m} F_{m, c}^{(r)}(x ; y) \quad(n \geq 0)
$$

where

$$
\begin{aligned}
a_{m} & =\frac{1}{m !} \sum_{k=0}^{n-m} \frac{1}{k !} \sum_{l=0}^{k}\left(\begin{array}{l}
r \\
l
\end{array}\right) l !(-y)^{l} T(k, l)\left\langle 1 \mid t^{m+k} B_{n}(x)\right\rangle \\
& =\sum_{k=0}^{n-m} \sum_{l=0}^{k}\left(\begin{array}{l}
r \\
l
\end{array}\right) l !(-y)^{l} T(k, l)\left(\begin{array}{c}
n \\
m
\end{array}\right)\left(\begin{array}{c}
n-m \\
k
\end{array}\right)\left\langle 1 \mid B_{n-m-k}(x)\right\rangle \\
& =\sum_{k=0}^{n-m} \sum_{l=0}^{k}\left(\begin{array}{l}
r \\
l
\end{array}\right) l !\left(\begin{array}{c}
n \\
m
\end{array}\right)\left(\begin{array}{c}
n-m \\
k
\end{array}\right)(-y)^{l} T(k, l) B_{n-m-k},
\end{aligned}
$$

where $B_{n}=B_{n}(0)$ are Bernoulli numbers. Thus, by (44) and (45), we get

$$
B_{n}(x)=\sum_{m=0}^{n} \sum_{k=0}^{n-m} \sum_{l=0}^{k}\left(\begin{array}{l}
r \\
l
\end{array}\right) l !\left(\begin{array}{c}
n \\
m
\end{array}\right)\left(\begin{array}{c}
n-m \\
k
\end{array}\right)(-y)^{l} T(k, l) B_{n-m-k} F_{m, c}^{(r)}(x ; y) .
$$


Assume that

$$
x^{n}=\sum_{m=0}^{n} a_{m} F_{m, c}^{(r)}(x ; y) \quad(n \geq 0),
$$

where

$$
\begin{aligned}
a_{m} & =\frac{1}{m !} \sum_{k=0}^{n-m} \frac{1}{k !} \sum_{l=0}^{k}\left(\begin{array}{l}
r \\
l
\end{array}\right) l !(-y)^{l} T(k, l)\left\langle 1 \mid t^{m+k} x^{n}\right\rangle \\
& =\frac{1}{m !} \sum_{k=0}^{n-m} \frac{1}{k !} \sum_{l=0}^{k}\left(\begin{array}{l}
r \\
l
\end{array}\right) l !(-y)^{l} T(k, l)(n)_{m+k}\left\langle 1 \mid x^{n-m-k}\right\rangle \\
& =\frac{1}{m !} \frac{1}{(n-m) !} \sum_{l=0}^{n-m}\left(\begin{array}{l}
r \\
l
\end{array}\right) l !(-y)^{l} T(n-m, l)(n)_{n} \\
& =\left(\begin{array}{l}
n \\
m
\end{array}\right) \sum_{l=0}^{n-m}\left(\begin{array}{l}
r \\
l
\end{array}\right) l !(-y)^{l} T(n-m, l) .
\end{aligned}
$$

Hence,

$$
x^{n}=\sum_{m=0}^{n}\left(\begin{array}{l}
n \\
m
\end{array}\right) \sum_{l=0}^{n-m}\left(\begin{array}{l}
r \\
l
\end{array}\right) l !(-y)^{l} T(n-m, l) F_{m, c}^{(r)}(x ; y) .
$$

\section{Conclusions}

Recently, the two variable Fubini polynomials were introduced by Kargin (see [4]) and the central Fubini polynomials associated with central factorial numbers of the second kind by Kim et al. (see [8]). In this paper, we considered two variable higher-order central Fubini polynomials as a 'central analogue' of two variable higher-order Fubini polynomials. We investigated some properties, identities, and recurrence relations for these polynomials by making use of generating functions and umbral calculus. In particular, we obtained various expressions for the two variable higher-order central Fubini polynomials and expressed them in terms of some families of special polynomials and vice versa.

\section{Funding}

The authors received no specific funding for this work.

\section{Competing interests}

The authors declare that they have no competing interests.

Authors' contributions

All authors contributed equally to this work. All authors read and approved the final manuscript.

\section{Author details}

'Department of Mathematics, Kwangwoon University, Seoul, Republic of Korea. ${ }^{2}$ Department of Mathematics, Sogang University, Seoul, Republic of Korea. ${ }^{3}$ Department of Mathematics, Pusan National University, Busan, Republic of Korea.

\section{Publisher's Note}

Springer Nature remains neutral with regard to jurisdictional claims in published maps and institutional affiliations.

Received: 1 February 2019 Accepted: 14 May 2019 Published online: 22 May 2019 


\section{References}

1. Cakić, N.P., El-Desouky, B.S., Milovanović, G.V.: Explicit formulas and combinatorial identities for generalized Stirling numbers. Mediterr. J. Math. 10, 57-72 (2013)

2. Carlitz, L., Riordan, J.: The divided central differences of zero. Can. J. Math. 15, 94-100 (1963)

3. Dere, R., Simsek, Y.: Applications of umbral algebra to some special polynomials. Adv. Stud. Contemp. Math. (Kyungshang) 22(3), 433-438 (2012)

4. Kargin, L.: Some formulae for products of Fubini polynomials with applications (2016). arXiv:1701.01023v1 [math. CA], cf. MR3622263

5. Kilar, N., Simsek, Y.: A new family of Fubini type numbers and polynomials associated with Apostol-Bernoulli numbers and polynomials. J. Korean Math. Soc. 54(5), 1605-1621 (2017)

6. Kim, D.S., Jang, G.-W., Kwon, H.-I., Kim, T.: Two variable higher-order degenerate Fubini polynomials. Proc. Jangjeon Math. Soc. 21(1), 5-22 (2018)

7. Kim, D.S., Kim, T.: Some identities of Bell polynomials. Sci. China Math. 58(10), 2095-2104 (2015)

8. Kim, D.S., Kwon, J., Dolgy, D.V., Kim, T.: On central Fubini polynomials associated with central factorial numbers of the second kind. Proc. Jangjeon Math. Soc. 21(4), 589-598 (2018)

9. Kim, T.: A note on central factorial numbers. Proc. Jangjeon Math. Soc. 21(4), 575-588 (2018)

10. Kim, T., Kim, D.S.: On $\lambda$-Bell polynomials associated with umbral calculus. Russ. J. Math. Phys. 24(1), 69-78 (2017)

11. Kim, T., Kim, D.S.: Degenerate central Bell numbers and polynomials. Rev. R. Acad. Cienc. Exactas Fís. Nat., Ser. A Mat. (2019). https://doi.org/10.1007/s13398-019-00637-0

12. Kim, T., Kim, D.S.: A note on central Bell numbers and polynomials. Russ. J. Math. Phys. 27(1) (2020)

13. Kim, T., Kim, D.S., Jang, G.-W., Kwon, J.: Extended central factorial polynomials of the second kind. Adv. Differ. Equ. 2019, 24 (2019). https://doi.org/10.1186/s13662-019-1963-1

14. Pyo, S.-S.: Some identities of degenerate Fubini polynomials arising from differential equations. J. Nonlinear Sci. Appl. 11(3), 383-393 (2018)

15. Roman, S.: The Umbral Calculus. Pure and Applied Mathematics, vol. 111. Academic Press, New York (1984)

16. Simsek, Y.: Identities on the Changhee numbers and Apostol-type Daehee polynomials. Adv. Stud. Contemp. Math. (Kyungshang) 27(2), 199-212 (2017)

17. Simsek, Y.: Computation methods for combinatorial sums and Euler type numbers related to new families of numbers. Math. Methods Appl. Sci. 40(7), 2347-2361 (2017)

18. Simsek, Y.: New families of special numbers for computing negative order Euler numbers and related numbers and polynomials. Appl. Anal. Discrete Math. 12, 1-35 (2018)

\section{Submit your manuscript to a SpringerOpen ${ }^{\circ}$ journal and benefit from:}

- Convenient online submission

- Rigorous peer review

Open access: articles freely available online

- High visibility within the field

- Retaining the copyright to your article

Submit your next manuscript at $\gg$ springeropen.com 